\title{
Eficacia de la Hidroxiapatita en la Cicatrización de Injertos Oseos e Implantes Dentales: Una Revisión Sistemática de la Literatura
}

\author{
Hydroxyapatite Effectiveness in the Healing of Bone and Implants Dental Implants: \\ a Systematic Review of the Literature
}

Álvaro Pereira G. \& Patricio Oliva M..

PEREIRA, G. Á. \& OLIVA, M. P. Eficacia de la hidroxiapatita en la cicatrización de injertos oseos e implantes dentales: Una revisión sistemática de la literatura. Int. J. Odontostomat., 10(3):373-380, 2016.

RESUMEN: La hidroxiapatita $(\mathrm{HA})$ es un componente mineral del hueso. Se ha comercializado la HA sintética o aloplástica en forma porosa o no porosa, densa y reabsorbible o no reabsorbible. Se utiliza como injerto óseo dada sus propiedades osteoconductivas y estimuladoras de proliferación celular generando así condiciones favorables para la regeneración tisular. El objetivo de éste trabajo es evaluar la efectividad del tratamiento con HA en cirugías de implantes dentales e injertos óseos. Se realizó una revisión sistemática. Se revisó ensayos clínicos sobre cicatrización y los injertos de HA sintética en Pubmed, Cochrane Library, Google Académico, Blackwell y Ebsco. Los criterios de selección son pacientes candidatos a tratamientos de defectos periodontales o rehabilitación con implantes dentales, sin alteraciones sistémicas de importancia. La estrategia se resume en los siguientes puntos: Identificación del problema, Identificación de términos de búsqueda, Búsqueda sistemática, Selección de artículos mediante utilización de guías de análisis crítico, Incorporación de la información al REVMAN®, Análisis de sesgos y Meta análisis. Al evaluar el metaanálisis se observa que la HA es efectiva para la cicatrización de injerto óseo. Cuando se omiten los estudios de bajo peso, la medida de resumen se mantiene en la HA. El tratamiento con HA en la cicatrización ósea y de implantes dentales resulta positivo y con una alta tasa de éxito en el largo plazo aunque su rango de efectividad varía con los materiales a los cuales ésta se asocie.

PALABRAS CLAVE: Hidroxiapatita, implantes dentales, defectos periodontales, cicatrización, elevación seno maxilar, cicatrización implantes.

\section{INTRODUCTION}

La necesidad de practicar cirugías adicionales y el poco abasto que han dado los bancos de hueso junto con los riesgos asociados de transmitir infecciones se configuran como las razones fundamentales de la generación de biomateriales sintéticos que puedan sustituir al injerto de hueso en cirugías reconstructivas (Gonzáles, 2005).

Actualmente, los injertos de hueso autólogo permanecen como el procedimiento más utilizado, pero al necesitar un sitio donante conlleva una morbilidad asociada (Holmes, 2013). Dentro de las otras opciones se encuentran los materiales aloplásticos como la HA y los xenoinjertos, que son aquellos derivados de otra especie, generalmente bovina (Holmes). Como la gran mayoría de los materiales aloplásticos no tienen una capacidad osteoinductiva, se usan en conjunto con injertos autólogos u otros métodos como la regeneración ósea guíada (ROG) o plasma rico en plaquetas (PRP) (Jimi et al., 2012).

La hidroxiapatita es un material biocerámico basado en fosfato de calcio. Cuando se implanta en un sitio óseo, se reabsorbe lentamente, dejando así una reserva de fosfato y calcio disponible para los procesos de biomineralización y regeneración ósea (Heinz, 2009). El proceso de regeneración de tejido óseo necesita 3 componentes claves: una señal osteoinductiva,

*Facultad de Odontología, Universidad del Desarrollo, Concepción, Chile. 
un sustrato que actúa como andamio para la nueva formación ósea y finalmente, células del huésped capaces de diferenciarse en células óseas (Mangano et al., 2003). Estudios in vitro indicaron que la HA es un fuerte estimulador del ligamento periodontal y que además, actúa como un estimulador de la proliferación celular, lo que está relacionado con la activación del receptor del factor de crecimiento epidérmico (EGFR), la MAP kinasa (ERK1/2) y la vía de la proteína kinasa $B$ (Kasaj et al., 2008).

En investigaciones histológicas recientes de biopsias en elevaciones de seno maxilar usando HA nanocristalina, se observó la generación de nuevo hueso formado, aunque en cantidades limitadas a los 3 meses y un nuevo hueso trabecular a los 6 meses (Heinemann et al., 2009).

Los efectos positivos de la HA se atribuyen a la alta respuesta angiogénica y biocompatibilidad del material, mejorando así, la reparación ósea (Pietruska et al., 2012).

El objetivo de este estudio fue evaluar la efectividad de la hidroxiapatita en la cicatrización de implantes dentales e injertos óseos mediante una revisión sistemática de la literatura con metaanálisis que responde a la siguiente pregunta de búsqueda: ¿Es eficaz el uso de la hidroxiapatita en la cicatrización de implantes dentales e injertos óseos?

\section{MATERIAL Y MÉTODO}

Se realizó una revisión sistemática de la literatura científica. Se efectuó un metaanálisis de la información obtenida en los artículos relacionados con hydroxiapatita que además incorporen en sus componentes cirugía de implantes dentales y defectos periodontales, tanto en mandíbulas como también en maxilares con elevación de seno maxilar e injertos óseos, se determinó además su efectividad en la cicatrización.

Aplicación de los Criterios de selección: Se incluyen estudios clínicos controlados con y sin aleatorización, ensayos clínicos en distintas fases (I, II, III y IV) además de ensayos clínicos de diseño de boca dividida. Los pacientes, fueron mayores de 18 años y con rehabilitación protésica o sometidos a injertos óseos, y donde se investiga la hidroxiapatita como tratamiento de regeneración ósea.
Se excluyen pacientes que incorporan el tratamiento combinado con terapias coadyuvantes o pacientes que presenten una falta de precisión en las respuestas

Estrategia de Búsqueda: La búsqueda se llevó a cabo por dos investigadores de forma independiente, fueron extraídos por un revisor directamente de los textos completos de los artículos. Un segundo investigador verificó de forma independiente los datos extraídos. Las discrepancias se resolvieron mediante la comprobación de la fuente y el debate de la metodología expuesta en ella hasta llegar a un consenso.

Se utilizaron diferentes bases de datos tales como:

a) El Registro Cochrane Central de Ensayos Controlados (CENTRAL)

b) MEDLINE (PubMed) (desde 2000 hasta el 30 de diciembre de 2012)

c) EMBASE (desde 2000 hasta el 17 de diciembre de 2012)

d) EBSCO (de 2000 a 22 de diciembre 2012),

e) Google Scholar (sin plazo)

f) el registro de ensayos clínicos de la Colaboración Cochrane sin restricción de lenguaje.

Se ejecuta una búsqueda en metabuscadores (www.google.com) donde se chequean posibles artículos adicionales no indexados en buscadores biomédicos (Fig. 1).

Extracción de información: Se utilizan términos de búsqueda utilizando los componentes de la pregunta de búsqueda como modelo estructural (Tabla I).

La variable de intervención primaria es Hidroxiapatita y como variables de intervención secundarias se plantearon: implantes dentales, defectos periodontales, elevación de seno maxilar, y a su vez, dentro de los mismos términos claves, se utilizaron vocablos en idioma inglés, como: hydroxyapatite, dental implant, dental implants, hydroxyapatite and dental implants, hydroxyapatite and periodontal. Se utilizaron también otros niveles de búsqueda como términos booleanos, tales como: AND y OR para encontrar relaciones entre los injertos óseos y la rehabilitación con implantes. Se aplicó la guía Consort® para el análisis crítico de cada artículo.

Análisis Estadístico: El proceso de análisis de la información de los artículos seleccionados se basó en los siguientes pasos: 


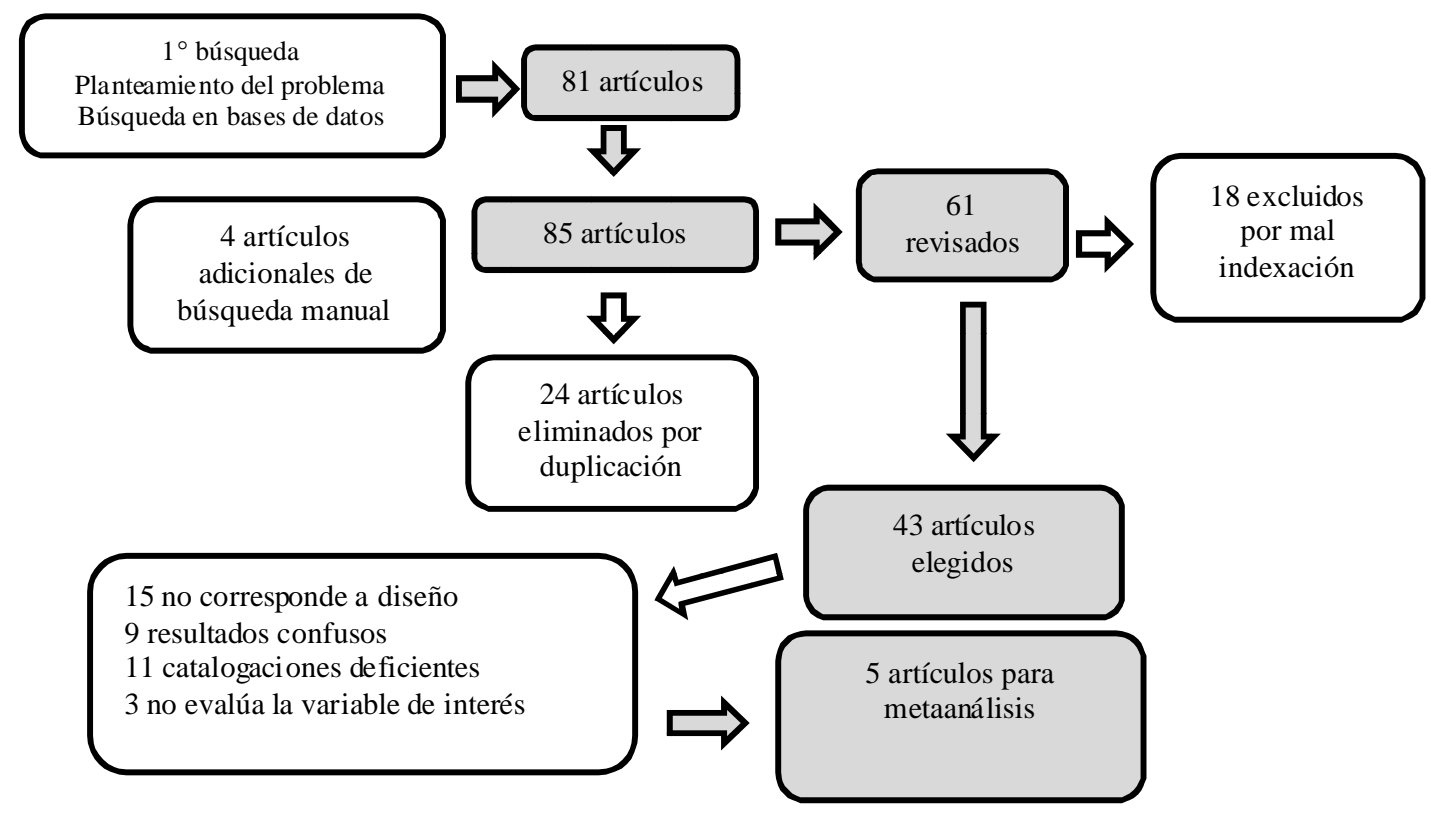

Fig. 1. Selección de los artículos.

Tabla I variables de la pregunta de investigación

\begin{tabular}{cccc}
\hline Pacientes & Intervención & Comparación & Outcome \\
\hline Pacientes sometidos a & Utilización de & No utilización del & Cicatrización \\
cirugías odontológicas & hydroxiapatita & compuesto & Seguridad \\
\hline
\end{tabular}

1. Descripción cualitativa de los estudios incluidos.

2. "Forest Plot" para la información de los resultados de sensibilidad y especificidad de los estudios individuales para cada método se combina con el tipo de diente y su respectiva superficie (RevMan Versión 5.1).

3. Se evaluó la calidad individual de los estudios a través QUADAS lista (RevMan versión 5.1).

4. Se buscó la presencia de sesgo de publicación mediante gráficos en embudo y sus respectivos intervalos de confianza (IC).

5. Se buscó la presencia de heterogeneidad (inconsistencia - I 2 ) de los estudios incluidos.

El análisis se realizó a través de la aplicación de un modelo aleatorizado. Se utiliza Mantel Haenszel para el estudio, ya que involucra estudios con muestras pequeñas y escasos eventos. La heterogeneidad se analiza mediante el Tau2 con un x2 además del 12 considerando los valores de 25,50 y $75 \%$ como bajo, moderada y alta heterogeneidad (L'abbé, 1994).
Para el análisis se opta por el modelo de efecto fijo, asumiendo que cualquier variación observada en las puntuaciones se deberá al error experimental de cada estudio particular, todo con una confiabilidad de un $95 \%$.

Cuando I2 es moderado, se establece un análisis de sensibilidad y se evalúa la causa del fenómeno. Si fuera bajo, se calcula el número necesario para tratar (NNT) para estimar el número necesario de sujetos para efectuar el análisis correspondientes (Pértega, 2005).

El análisis de sesgo se efectúo por dos autores que extrajeron la información y la cotejaron de forma independiente evaluando la calidad metodológica y el riesgo de sesgo.

La evaluación del riesgo de sesgo fue una evaluación en seis dominios:

1. Generación de la secuencia.

2. Ocultamiento de la asignación, que implica 
3. El cegamiento,

4. Seguimiento y exclusiones,

5. Informe selectivo de los resultados

6. Otro sesgo

\section{RESULTADOS}

El resultado de la búsqueda sistemática y análisis crítico de la información implicaron 5 artículos científicos, sumando una cantidad de 189 pacientes Los estudios se ordenan bajo la siguiente lógica como se puede ver en Tabla II.

Riesgo de Sesgo. La evaluación del riesgo de sesgo en los artículos seleccionados se estableció en seis dominios específicos y que cuyo objeto es develar la calidad de la información contenida en las investigaciones incorporadas al metaanálisis, necesaria para la toma de decisiones (Tabla III):

La hidroxiapatita en injertos óseos presentó los siguientes resultados: a) peso: sólo dos estudios (Pietruska et al. \& Schwarz et al. 2009) poseen un peso que supera el $20 \%$, existiendo 2 estudios con que tienen un peso inferior al $10 \%$. B) OR: entendido como el cociente de dos razones, el numerador es la razón de la probabilidad de que un evento suceda y la probabilidad de que no suceda bajo ciertas condiciones y el denominador es la razón de la probabilidad de que dicho evento suceda y la probabilidad de que no suceda bajo las condiciones complementarias (Fig. 2).

El metaanálisis presentó una heterogeneidad (I2) del $0 \%$, con una significatividad del 0,00 (menor a $0,05)$, lo cual implica una muy baja dispersión de los resultados de los estudios. Dos estudios (Heinz et al. \& Prosper et al., 2003), poseen peso bajo y un intervalo de confianza amplio, situándose en el control. La evaluación de resumen del metaanálisis demuestra que la Hidroxiapatita es efectiva para la cicatrización en injerto óseo.

Análisis de Sensibilidad. El análisis de sensibilidad implica estudiar la influencia que posee cada uno de los estudios en la estimación global del efecto y con ello evaluar la robustez de la medida obtenida. Cuando se eliminaron los estudios que poseían un bajo peso (bajo el $10 \%$ ) y cuyos intervalos de confianza son amplios, se expresan variaciones en los resultados del metaanálisis (Fig. 2), con un OR final de 0,70 con intervalos de 0,25 y 1,99 y una heterogeneidad (I2) que se mantiene en $0 \%$. La medida de resumen se mantuvo en la HA. (Fig. 3)

Tabla II. Resumen de estudios incluídos.

\begin{tabular}{ccl}
\hline Estudio incluído & \multicolumn{1}{c}{ Intervención } & \multicolumn{1}{c}{ Outcome } \\
\hline Mangano et al. , 2007 & Ensayo clínico comparativo & La hidroxiapatita bovina y la HA porosa sintética se pueden usar \\
& satisfactoriamente como injertos óseos para elevaciones de seno.
\end{tabular}

Schwarz et al., 2009.

Pietruska et al. , 2012.

Ensayo clínico ramdomizado, con seguimiento clínico de 4 años, año 2009.

Ensayo clínico ramdomizado con seguimiento de 1 año.

Ensayo clínico ramdomizado.

Heinz et al., 2009.

Prosper et al., 2003.
Ensayo clínico ramdomizado con seguimiento clínico de 4 años.
Mientras que la aplicación de NBM + CM resultó en mejoras clínicas a lo largo de 4 años, los resultados obtenidos a largo plazo con NHA sin membrana de barrera deben considerarse como pobres.

El uso adicional de nano hidroxiapatita como injerto óseo luego $d \epsilon$ una cirugía a colgajo abierto no mejora los resultados clínicos $n$ radiográficos obtenidos.

Después de 6 meses de la cirugía, el tratamiento de defectos infraóseos con una técnica de preservación de tejidos blandos y la aplicación de NHA lleva a mejorar los resultados clínicos del sondaje del surco y de la altura ósea comparado a la técnica de preservaciór de tejidos blandos sola.

Los implantes colocados en combinación con un material aloplásticc reabsorbible o con una membrana reabsorbible, dan resultados previsibles a la rgo plazo cuando se r estauran con prótesis fijas plurales. 
Tabla III. Análisis de sesgo.

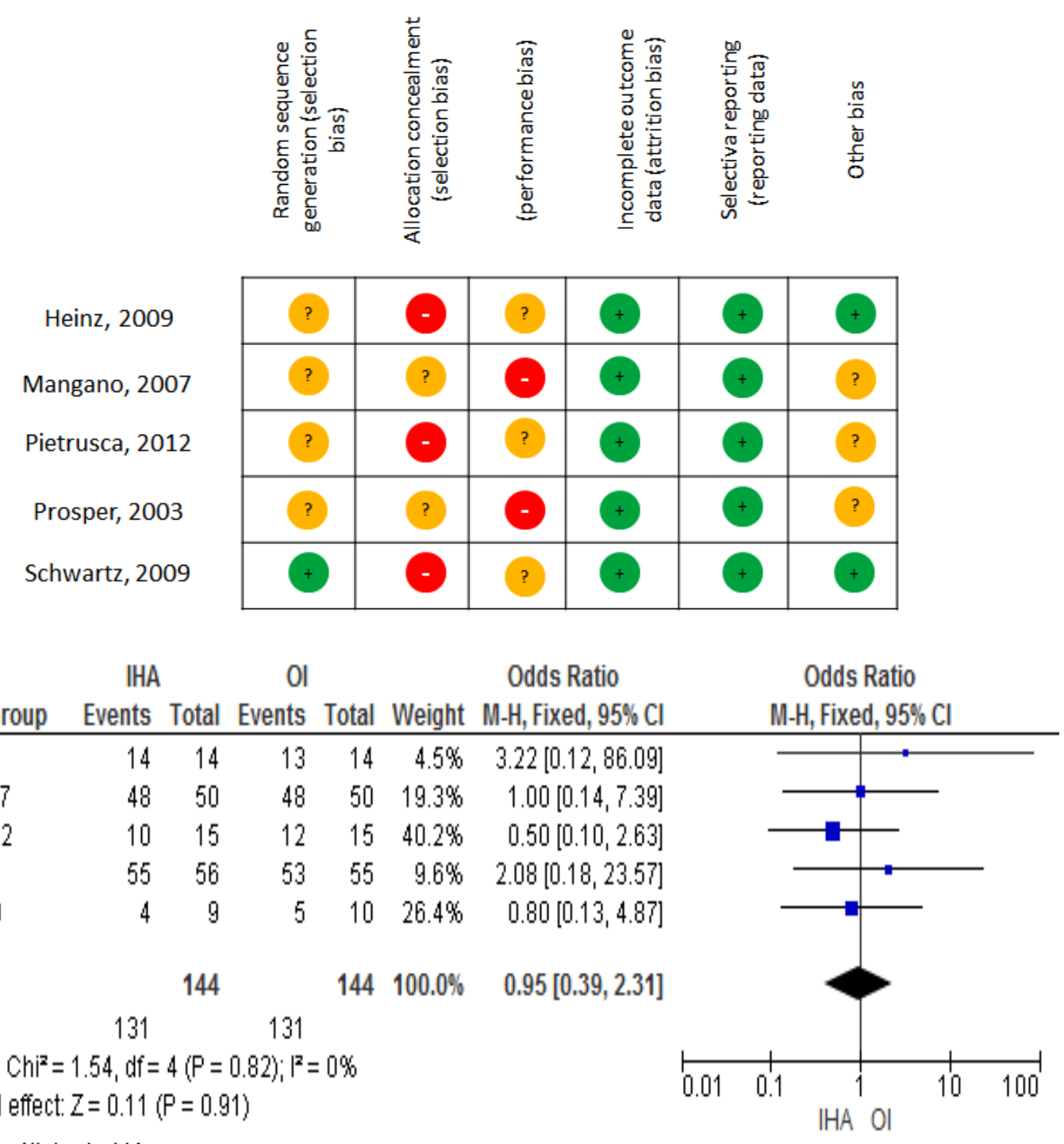

Fig. 2. Metaanálisis de HA.

IHA Ol Odds Ratio Odds Ratio

Study or Subgroup Events Total Events Total Weight M-H, Fixed, $95 \% \mathrm{Cl}$

$\begin{array}{llllllll}\text { Heinz } 2009 & 14 & 14 & 13 & 14 & 0.0 \% & 3.22[0.12 & 86.09]\end{array}$

$\begin{array}{lllllll}\text { Mangano, } 2007 \quad 48 & 50 & 48 & 50 & 22.5 \% & 1.00[0.14,7.39]\end{array}$

Pietruska, 2012

$\begin{array}{llllll}10 & 15 & 12 & 15 & 46.8 \% & 0.50[0.10,2.63]\end{array}$

Prosper, 2003

$\begin{array}{llllll}55 & 56 & 53 & 55 & 0.0 \% & 2.08[0.18,23.57]\end{array}$

Schwarz, 2009

$\begin{array}{llllll}4 & 9 & 5 & 10 & 30.8 \% & 0.80[0.13,4.87]\end{array}$

Total $(95 \% \mathrm{Cl})$

74

$75 \quad 100.0 \%$

$0.70[0.25,1.99]$

Total events

62 65

Heterogeneity: $\mathrm{Chi}^{2}=0.30, \mathrm{df}=2(\mathrm{P}=0.86) ; \mathrm{I}^{2}=0 \%$

Test for overall effect: $Z=0.66(P=0.51)$

M-H, Fixed, $95 \% \mathrm{Cl}$

Fig. 3. Análisis de Sensibilidad.

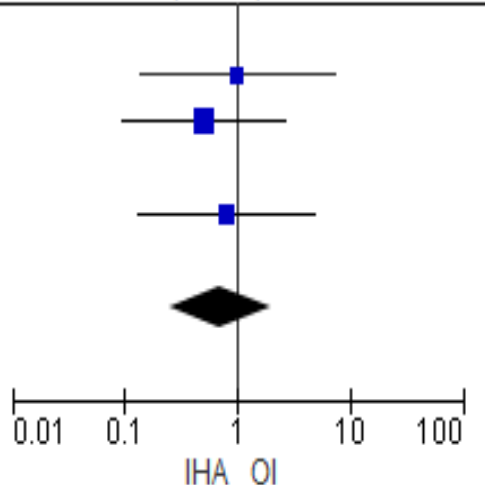




\section{DISCUSIÓN}

En el caso de preservación de reborde en alvéolos con defectos óseos de más de $5 \mathrm{~mm}$ en la pared bucal, la HA puede disminuir la reabsorción de la cresta alveolar luego de la extracción dentaria, resultando en una mejor regeneración horizontal de la pared bucal (Sisti et al., 2012) lo que coincide con los resultados obtenidos por Prosper et al.(2003), quien evaluó el uso de la HA en alveólos post- extracción para luego rehabilitar con implantes, obteniendo un éxito de $98,2 \%$ a los 4 años. Esto es muy útil en casos donde se rehabilitará de manera fija un diente extraído, ya sea con implante o con prótesis fija, ya que, se puede disminuir la inminente reabsorción ósea que se produce luego de la extracción.

Se utilizó como tratamiento de peri-implantitis donde la eficacia del injerto va a depender en gran medida del tipo de implante y de las distintas superficies de estos. Se consiguió disminuir la profundidad del sondaje y aumentar el nivel de inserción clínica pero, esto último, luego de 24 meses comenzó a disminuir, lo que se podría explicar por acumulación de placa en la zona que provoca una inflamación en los tejidos peri-implantarios (Schwarz et al., 2006).

Por otra parte, se ha usado la HA para el tratamiento de lesiones de furca mandibulares donde se consiguió una mejora muy significativa frente a la terapia de destartraje manual con colgajo pero es de mucha importancia señalar que la respuesta clínica a terapias regenerativas está condicionada por parámetros pre-quirúrgicos tales como discrepancias en los protocolos de tratamiento, acumulación de placa, inflamación gingival y la morfología de la lesión de furca entre otros, por lo que todos estos parámetros se deben tener en cuenta al momento de realizar y evaluar este tipo de intervenciones ya que, podrían modificar tanto negativa como positivamente los resultados finales (Santana et al., 2009). La adición de fosfato tricálcico beta y de plasma rico en plaquetas a la HA, demostró mejorar significativamente la regeneración periodontal debido a diversas propiedades positivas del PRP como el ser un injerto autológo, biológicamente aceptable, promover la angiogénesis y sus propiedades hemostáticas entre otras, por lo que el uso combinado de estos materiales de injerto debería ser estudiado más a fondo (Kaushick et al., 2011).
Otro uso que se observó en los injertos de HA fue las elevaciones de seno maxilar para asistir la cicatrización ósea y generar así un reborde y alveólo óseo óptimo para la colocación de implantes. En el estudio de Mangano et al. (2007), a los 6 meses se observó aposición ósea alrededor de la HA y también se vieron signos de osteoinducción, algo que no se observó en otros estudios de esta revisión. Esto concuerda con los resultados de los estudios de Mazor et al. (2000) y Strietzel et al. (2007), en el que se observó que el porcentaje de área de HA remanente disminuía desde la periferia hacia el centro donde se iba remplazando por nuevo tejido óseo estableciendo un nuevo sitio, tanto cuantitativa como cualitativamente apto para poder lograr una correcta estabilidad primaria, oseointegración y posterior rehabilitación protésica. Con respecto a la seguridad del tratamiento, encontramos una alta tasa de éxito de implantes en el largo plazo y sin efectos secundarios pero, con resultados variables ya que, al asociar la HA con otros materiales como PRP, injertos autólogos o barreras de membrana, entre otros, se obtienen efectos positivos aunque, en distintas medidas. La importancia clínica del uso de injertos de HA radica en su biocompatiblidad y beneficios al asociarlos con otros tipos de injertos para conseguir así mayor densidad y volumen óseo, otorgando unas mejores condiciones funcionales y/o estéticas para el tratamiento rehabilitador protésico o periodontal. En casos donde no se puede recurrir a una cirugía adicional para extraer tejido óseo desde otra zona, el uso de materiales sintéticos es de mucha ayuda para conseguir un relleno óseo adecuado para el implante o para los tejidos blandos.

Recomendación. La hidroxiapatita sintética resulta ser una alternativa de tratamiento como coadyuvante en la cicatrización ósea. Los estudios demostraron su capacidad osteoconductiva y de estimular la proliferación celular mejorando así la calidad ósea para terapias periodontales, elevaciones de seno o rehabilitaciones sobre implante. Pero aún faltan estudios que señalen de manera específica los mecanismos de acción de la HA sobre la cicatrización tisular y el tipo, cantidad y calidad ósea obtenida para poder comparar con otras terapias regenerativas y permitir así al tratante tomar una decisión más informada sobre los beneficios de las distintas terapias disponibles. 
PEREIRA, G. Á. \& OLIVA, M. P. Hydroxyapatite effectiveness in the healing of bone and implants dental implants: A systematic review of the literature. Int. J. Odontostomat., 10(3):373-380, 2016.

ABSTRACT. Hydroxyapatite $(\mathrm{HA})$ is a mineral component of bone. Hydroxyapatite has been marketed as synthetic or alloplastic, porous or nonporous, dense and resorbable or non-resorbable form. It is used as bone graft due to its osteoconductive properties and stimulation of cell proliferation, generating favorable conditions for tissue regeneration. The aim of this study is to evaluate the effectiveness of treatment with HA in dental implant surgeries and bone grafts. We conducted a systematic review. Clinical trials on healing and graft of synthetic hydroxyapatite were reviewed in PubMed, Cochrane Library, Google Scholar, Blackwell and Ebsco. The selection criteria was: patient candidates for treatment of periodontal defects or rehabilitation with dental implants without significant systemic abnormalities. The strategy is summarized in the following: Identification of the problem, identifying search terms, systematic search and selection of articles by using critical analysis guides, Incorporating REVMAN ${ }^{\circledR}$ information, unbiased analysis, and Meta-analysis. Assessing the metaanalysis shows that the HA is effective for healing bone graft. When omitting low weight studies, the summary measure remains in the HA. Treatment with HA in bone healing and dental implants, is positive with a high rate of success in the long term, although its effective range varies according to the materials to which it is associated. healing.

KEY WORDS: hidroxiapatite, dental implants, periodontal defects, healing, maxillary sinus elevation, implant

\section{REFERENCIAS BIBLIOGRÁFICAS}

Gonzáles, R. Hidroxiapatita Porosa Coralina Hap-200. 15Años de Aplicaciones Clínicas. Revista CENIC Ciencias Biológicas, 36, 2005

Heinz, B.; Kasaj, A.; Teich, M.; Jepsen, S. Clinical effects of nanocrystalline hydroxyapatite paste in the treatment of intrabony periodontal defects: a randomized controlled clinical study. Clin. Oral Investig., 14:525-31, 2009.

Holmes, J. Considerations in Dental Implant Placement in the Young Patient: A Surgeon's Perspective. Seminars in Orthodontics, 19(1):24-36, 2013.

Jimi, E.; Hirata, S.; Osawa, K.; Terashita, M.; Kitamura, C.; Fukushima, $\mathrm{H}$. The Current and Future Therapies of Bone Regeneration to Repair Bone Defects. Int. J. Dentistry, 12:1-7, 2012.

Kasaj, A.; Röhrig, B.; Zafiropoulos, G.G.; Willershausen, B. Clinical evaluation of nanocrystalline hydroxyapatite paste in the treatment of human periodontal bony defects-a randomized controlled clinical trial: 6-month results. J. Periodontol., 79(3):394400, 2008.

Kaushick, B.T.; Jayakumar, N.D.; Padmalatha, O.; Varghese, $S$. Treatment of human periodontal infrabony defects with hydroxyapatite $+b$ tricalcium phosphate bone graft alone and in combination with platelet rich plasma:A randomized clinical trial. Indian J. Dent. Res., 22(4):505-10, 2011.

Mangano, C.; Bartolucci, E.; Mazzocco, C. A new porous hydroxyapatite for promotion of bone regeneration in maxillary sinus augmentation: Clinical and histologic study in humans. Int. J. Oral Maxillofac. Implants, 18:23-30, 2003.
Mangano, C.; Scarano, A.; Perrotti, V.; lezzi, G. \& Piattelli. A. Maxillary sinus augmentation with a porous synthetic hydroxyapatite and bovine-derived hydroxyapatite: a comparative clinical and histologic study. Int. J. Oral Maxillofac. Implants, 22(6):980-6, 2007.

Mazor, Z.; Peleg, M.; Garg, A.; Chaushu, G. The Use of Hydroxyapatite Bone Cement for Sinus Floor Augmentation with Simultaneous Implant Placement in the Atrophic Maxilla. A Report of 10 Cases. J. Periodontology, 71(7):1187-94, 2000

Pietruska, M., Skurska, A., Pietruski, J., Dolinska, E., Arweiler, N., Milewski, R., Duraj, E., Sculean, A. Clinical and radiographic evaluation of intrabony periodontal defect treatment by open flap debridement alone or in combination with nanocrystalline hydroxyapatite bone substitute. Ann. Anatomy, 2012.

Prosper, L.; Gherlone, E.; Redaelli, S.; Quaranta, M. Four-year follow-up of larger-diameter implants placed in fresh extraction sockets using a resorbable membrane or a resorbable alloplastic material. Int. J. Oral Maxillofac. Implants, 18(6):856-64, 2003.

Rajesh, J.B.; Nandakumar, K.; Varma, H.K.; Komath, M. Calcium phosphate cement as a "barrier-graft" for the treatment of human periodontal intraosseous defects. Indian J. Dent Res., 20(4):471-9, 2009.

Santana, R.; Mattos, C.; Van Dyke, T. Efficacy of combined regenerative treatments in mandibular class II furcation defects. J. Periodontol., 80(11):1756-64, 2009. 
Schwarz, F.; Bieling, K.; Latz, T.; Nuesry, E. \& Becker, J. Healing of intrabony peri-implantitis defects following application of a nanocrystalline hydroxyapatite (Ostim ${ }^{\mathrm{TM}}$ ) or a bovine-derived xenograft (Bio-Oss ${ }^{\mathrm{TM}}$ ) in combination with a collagen membrane (Bio-Gide ${ }^{\mathrm{TM}}$ ). A case series. J. Clin. Periodontol., 33:491-9, 2006.

Schwarz, F.; Sahm, N.; Bieling, K.; Becker, J. Surgical regenerative treatment of peri-implantitis lesions using a nanocrystalline hydroxyapatite or a natural bone mineral in combination with a collagen membrane: a four-year clinical follow-up report. J. Clin. Periodontol., 36(9):80714, 2009.

Sisti, A.; Canullo, L.; Mottola, M.; Covani, U.; Barone, A.; Botticelli, D. Clinical evaluation of a ridge augmentation procedure for the severely resorbed alveolar socket: multicenter randomized controlled trial, preliminary results. Clin. Oral Impl., 23(5):526-35, 2012.

Strietzel, F.P.; Reichart, P.A.; Graf, H.L. Lateral alveolar ridge augmentation using a synthetic nano-crystalline hydroxyapatite bone substitution material (Ostims). Preliminary clinical and histological results. Clin. Oral Impl., 18(6):743-51, 2007.

Trombelli, L.; Franceschetti, G.; Rizzi, A.; Minenna, P.; Minenna, L.; Farina, R. Minimally invasive transcrestal sinus floor elevation with graft biomaterials. A randomized clinical trial. Clin. Oral Impl., 23(4):424-32, 2012.

Heinemann, F.; Mundt, T.; Biffar, R.; Gedrange, T.; Goetz, W. A 3-year clinical and radiographic study of implants placed simultaneously with maxillary sinus floor augmentations using a new nanocrystalline hydroxyapatite. J. Physiol. Pharmacol., 60 (8):91-7, 2009.
Dirección para correspondencia

Patricio Olivo

Facultad de Odontología

Universidad del Desarrollo

Concepción

Barros Arana 1734

Concepción

CHILE

Email: alvaropereirag1@gmail.com

Recibido : 09-01-2015

Aceptado: 03-10-2016 